lab diagnosed) and were treated with multi-dose Mtz, 96\% reported taking all their medicine. While $36 \%$ of these women reported condomless sex during follow-up, there was no association between sexual exposure and BV status at TOC. Of these 46 women, $42.9 \%$ remained $\mathrm{BV}+$ at TOC and $19.4 \%$ reported BV-related symptoms. BV status at TOC was not associated with TV cure rates $(p>0.56)$.

Conclusion A high rate of BV co-infection (49.8\%) was found among women with TV, much of which was asymptomatic. The rate of BV persistence post multi-dose Mtz was also high both microbiologically (42.9\%) and clinically (19.4\%) and did not appear to be influenced by TV treatment status. Additional research and development of novel therapeutics (i.e. biofilm disruptors) are urgently needed for women with BV, particularly among TV+ women where BV rates are high.

\section{P2.34 DOES ORAL SEX CAUSE FEMALE INFERTILITY?}

Atrik M Bavoil, Patricia X Marques, Rebecca Brotman, Jacques Ravel. University of Maryland, Baltimore, USA

\subsection{6/sextrans-2017-053264.210}

Introduction We present the following tri-factorial hypothesis:

1. All members of the Chlamydiaceae have evolved primarily as commensals of the digestive tract of their host(s) with fecal-oral transmission (FOT) as the principal route of dissemination to new hosts. In communities where fecal-oral transmission is reduced (e.g., via global sanitation), the occurrence of chlamydiae in the digestive tract of their host is reduced.2. Chlamydia trachomatis is a commensal microorganism of the human gastro-intestinal (GI) tract, and an opportunistic pathogen in the genital and respiratory tracts, and the conjunctiva. Under conditions of reduced FOT, direct contact (e.g., sexual) is the primary mode of transmission.

3. C. trachomatis is efficiently transmitted to the GI tract of new hosts via oral sex. The practice of oral sex has 'reintroduced' C. trachomatis to the human GI tract in communities where FOT was previously reduced.

Methods Circumstantial, historical and recent evidence from humans and animals that support the hypothesis is reviewed. Imaging of mCherry expressing Chlamydia muridarum in the murine GI tract was obtained.

Results and Conclusion: Tenets 1 and 2 imply a paradigm shift to reflect a revision of the status of C. trachomatis from that of a principal pathogen to that of a commensal organism that causes opportunistic infection at mucosal epithelia other than its preferred GI site. High frequency on/off switching of the expression of autotransported polymorphic membrane proteins, the unique properties of peptidoglycan and lipo-oligosaccharide, and observed extruded inclusions in the GI tract of C. muridarum-infected mice may facilitate chlamydial survival and colonisation of the GI tract.Tenet 3 implies that orally inoculated chlamydiae that survive in and colonise the GI tract, may reach the rectum and chronically, or episodically, infect the female genital tract eventually causing/contributing to tubal pathology and infertility. The global hypothesis therefore raises the provocative question: does oral sex cause or contribute to female infertility?
NEUROSYPHILIS: STILL THE SHADOW ON EARTH

Pingyu Zhou; Shanghai Skin Disease Hospital, Popular Republic of China

\subsection{6/sextrans-2017-053264.211}

Treponema pallidum subsp. pallidum (T. pallidum), the etiologic agent of syphilis, can disseminate into virtually any organ including the central nervoussystem (CNS). Among those infected organs, the diagnosis and treatment of central nervous system (CNS) infection is recognised to be the most challenging. If left untreated, neuroinvasion with $T$. pallidum might lead to asymptomatic meningitis and develop severe even irreversible symptomatic neurosyphilis (NS). In the early 20 th century, about $10 \%$ of the population of the United States and Europe were infected with syphilis. A century has passed, syphilis still remains a global public health problem, especially in developing countries, such as China, where an estimated 400 thousand people were infected annually in recent ten years.Despite a major health consequence that can cause undue physical, psychological harm and suffering for patients, neurosyphilis has not yet been a priority and remains a medical and public-health problem in many countries. The reasons that the surveillance data of neurosyphilis are limited at global level may because of the following: 1 . the $\mathrm{Tp}$ invades the CNS in most patients with early syphilis, who may have no symptom at all, which is difficult to determine which patient requires lumbar puncture (LP); 2. the diagnosis of neurosyphilis relies on CSF findings and the laboratory test criteria for diagnosis of neurosyphilis is neither sensitive nor specific; 3.symtomatic neurosyphilis is a "great imitator" and lack of specific clinical manifestations, which may result in misdiagnosis and leaves the disease without treatment for years. Here we reviewed the neurosyphilis in Shanghai Skin Disease hospital, China main land and investigated more than 6000 syphilis patients in Shanghai Skin Disease Hospital. We reinstate the need for LP among syphilis patients, particularly in the settings where syphilis is prevalent among key populations. As the incidence of syphilis continues to increase, further work is needed to better understand neurosyphilis.

\section{P2.36 SEXUALLY TRANSMITTED CO-INFECTIONS AND THE EFFECT OF DRUG USE ON RISK OF VIROLOGIC FAILURE AMONG HIV-POSITIVE MEN ON ANTIRETROVIRAL THERAPY}

${ }^{1}$ Ramandip Grewal, ${ }^{2}$ Vanessa Allen, ${ }^{3}$ Ahmed M Bayoumi, ${ }^{4}$ Sandra L Gardner, ${ }^{3}$ Rupert Kaul, ${ }^{2}$ Tony Mazzulli, ${ }^{5}$ Frank Mcgee, ${ }^{1}$ Veronika Moravan, ${ }^{3}$ Tyler O'neill, ${ }^{6}$ Janet Raboud, ${ }^{7}$ Sean B Rourke, 'Darrell HS Tan, 'Ann N Burchell. 'St. Michael's Hospital, Toronto, Canada; ${ }^{2}$ Public Health Ontario, Toronto, Canada; ${ }^{3}$ University of Toronto, Toronto, Canada; ${ }^{4}$ Baycrest Health Science, Toronto, Canada; ${ }^{5}$ Ontario Ministry of Health And Long Term Care, Toronto, Canada; ${ }^{6}$ University Health Network, Toronto, Canada; ${ }^{7}$ Ontario HIV Treatment Network, Toronto, Canada

\subsection{6/sextrans-2017-053264.212}

Introduction Incidence of syphilis, chlamydia and gonorrhoea continue to rise among HIV-positive men who have sex with men (MSM) in Ontario. We previously observed an elevated risk of sexually transmitted infections (STI) among recreational drug users. Our aim was to determine the effect of a new STI diagnosis and recreational drug use on virologic failure (VF) among MSM successfully treated with antiretroviral 